\title{
SHORT AND LONG LATENCY JAW-OPENING REFLEX RESPONSES ELICITED BY MECHANICAL STIMULATION IN MAN
}

\author{
Y. Yamada*, C. S. Stohler $\dagger$, K. Shimada* and M. M. Ash JR $\dagger$ \\ *Department of Physiology, School of Dentistry, Niigata University, Niigata 951, Japan and $\uparrow$ Department \\ of Occlusion, School of Dentistry, University of Michigan, Ann Arbor, MI 48109, U.S.A.
}

\begin{abstract}
Summary - Jaw-opening reflex responses elicited by tapping the chin during maximum clenching in incisal edge-to-edge contact position were studied in 10 healthy subjects. Stimuli were also delivered during weak clenching on a rubber stamp, separating the incisors by approx. $10 \mathrm{~mm}$ and protruding the mandible to the edge-to-edge incisor relationship. All four central incisors were stimulated simultaneously. With weak stimuli, there was a short-latency $(9.5 \mathrm{~ms})$ digastric response which may have had a disynaptic pathway. Taps of moderate strength produced long-latency $(20 \mathrm{~ms})$ responses, and sometimes a short-latency $(9.5 \mathrm{~ms})$ component as well. Strong (non-painful) taps produced an even longer-latency digastric response, $30 \mathrm{~ms}$ or more following the stimulus with less synchronization than earlier responses. Jaw-jerk reflexes occurred $8.5 \mathrm{~ms}$ following the tap, independently of the magnitude of the stimulus. Local anaesthesia of the upper and lower incisors abolished the digastric muscle response. Thus large periodontal afferents may be responsible for the early digastric reflex activity and smaller fibres for later effects. Temporal summation of the reflex response probably occurred when all incisors were stimulated simultaneously.
\end{abstract}

\section{INTRODUCTION}

Many studies have provided evidence that mechanical stimulation to the periodontal apparatus induces facilitatory or inhibitory responses of jaw-opening and jaw-closing muscles (Hannam and Matthews, 1969; Sessle and Greenwood, 1976; Sessle, 1977a; Nakamura et al., 1982). Detailed description of the jaw-opening reflex pathway in cats are available (Kidokoro et al., 1968; Sumino, 1971; Junge, Faermark and Friedman, 1979). Evidence is mainly based upon research in animals, e.g. cats or rabbits. Data for man are scarce and raise some doubt about the existence of a jaw-opening reflex in man (Beaudreau, Daugherty and Masland, 1969; Gillings and Klineberg, 1975). However, such reflex activity with a latency of $28 \mathrm{~ms}$, coinciding with the termination of the silent period of the masseter muscle, has been reported by Yamada and Ash (1982). As stressed by Thexton (1974), the study of reflexes should be performed using mechanical rather than electrical stimuli as these resemble the natural circumstances. Investigations under static and dynamic conditions require to be distinguished. Whereas the reflex behaviour under static observation may vary with the experimental condition, the reflex response during mastication shows more plasticity and appears to be highly complex (Hannam and Lund, 1981). The view that the jaw-opening response is a protective reflex to a noxious stimulus is based upon the observation, that the digastric reflex response is associated with a higher threshold compared with facial contractions (Keller, Vyklicky and Sykova, 1972). Our purpose was to investigate the jaw-opening reflex in man under static jaw conditions using various stimulus levels.

\section{MATERIALS AND METHODS}

\section{Experimental subjects}

The experiments were performed on ten male students, aged 21 to 28 years, with complete, natural dentitions.

\section{Electromyography}

Bipolar electromyographic recordings were made from the right masseter and right digastric muscles. $\mathrm{Ag} / \mathrm{AgCl}$ disc electrodes were applied. Surface electrodes were placed in the direction of main massetermuscle fibres, $20 \mathrm{~mm}$ apart centre-to-centre. For the anterior belly of the digastric muscle, electrodes were placed $20 \mathrm{~mm}$ apart parallel to the muscle fibre direction. The anterior electrode was attached to the area over the insertion of the anterior belly into the mandible (Hannam, Matthews and Yemm, 1968). An ear-clip electrode, placed on the right ear lobe, was used as a ground reference. Subjects performed voluntary open-close movements to confirm that the electrodes were sampling appropriate signals.

\section{Clinical procedure}

Subjects were seated upright in a dental chair with a conventional headrest. Reflex responses were elicited by tapping on the chin during maximum clenching in incisal edge-to-edge contact position. Stimuli were further delivered when weakly clenching on a rubber stamp, separating the upper and lower incisors by approx. $10 \mathrm{~mm}$ and protruding the mandible to the incisal edge-to-edge configuration (Fig. 1). In this second condition, all four central incisors were stimulated simultaneously using weak, moderate and strong (non painful) mechanical taps to the rubber 

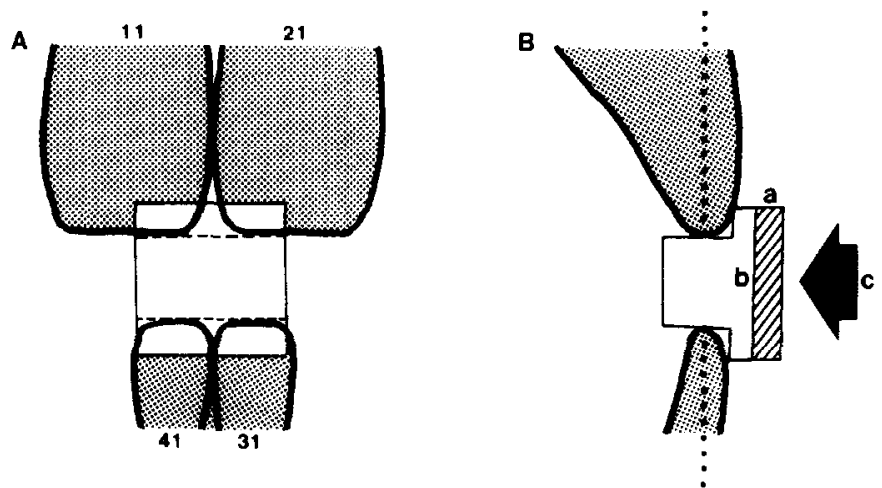

Fig. 1. Tap stimulus was applied to all four central incisors using a rubber stamp (b) which separated upper and lower teeth approx. $10 \mathrm{~mm}$. A piezo-electric sensor (a) was used for the measurement of the magnitude of the tap stimulus (c).

stamp. The magnitude of the stimulus was measured using a piezo-electric device placed in the rubber stamp and monitored on a storage oscilloscope. Experiments were repeated after careful local anaesthesia of upper $(1.5 \mathrm{ml})$ and lower incisors $(1.5 \mathrm{ml}$, 2 per cent xylocaine).

\section{Analysis}

Bipolar, surface electromyographic signals (EMG) were amplified with a frequency response approximately linear from $30 \mathrm{~Hz}$ to $3 \mathrm{kHz}$, and recorded using a FM tape recorder. After digitizing at a sampling rate of $400 \mu \mathrm{s}$ per sample, the EMG signal was fully rectified and averaged by triggering on the hammer blow. Signal averaging was done on ten responses from the trigger point to $100 \mathrm{~ms}$ after the trigger. The results were normalized to the maximum amplitude and plotted by an $\mathrm{x}-\mathrm{y}$ plotter under microcomputer control. Ten raw EMG signals were superimposed.

\section{RESULTS}

\section{Artifact}

As the stimulis was a mechanical tap, some impact of the vibration on the electromyogram may be expected, especially in jaw-opening muscle recordings. Such interference is likely to occur when surface electrodes are applied. Figure 2 shows an example in a digastric electromyogram, obtained with loose electrode wires, which only lasted for approx. $5 \mathrm{~ms}$ and could therefore easily be distinguished from a reflex response. No artifact was seen in the masseter muscle or when wires were firmly secured.

Tap on chin during maximum clenching in edge-toedge contact position

Tap stimulus was delivered on the chin during maximum clenching in edge-to-edge contact position. A clear response was observed in the digastric muscle activity about $24.5 \mathrm{~ms}$ after the tap stimulus. A smaller response was also found shortly after the stimulus. Its latency was about $10 \mathrm{~ms}$ and close to that of the jaw-jerk reflex in the masseter electromyogram (Fig. 3). The long-latency response was found consistently in all the subjects, the shortlatency response in only two subjects.

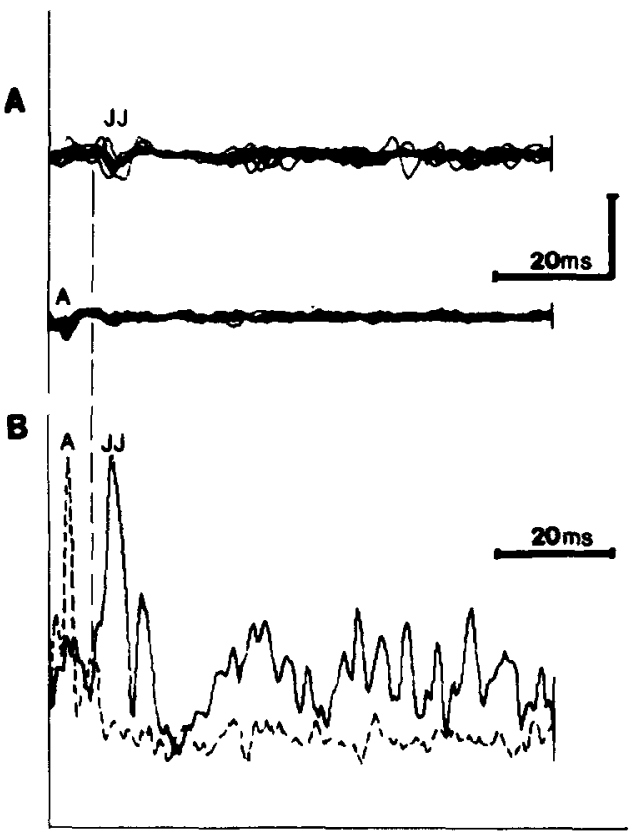

Fig. 2. Artifacts. (A) Superimposition of 10 raw electromyograms of the masseter (upper) and digastric (lower) muscles. (B) Averaged and normalized response of 10 stimuli delivered to the incisors while subject was clenching in intercuspal edge-to-edge configuration. Graph was generated from recordings displayed in $\mathrm{A}$. The masseter activity is indicated by smooth, the digastric activity by broken lines. Calibrations: vertical bar equals 0.1 and $0.05 \mathrm{mV}$ for the masseter and digastric muscle, respectively.

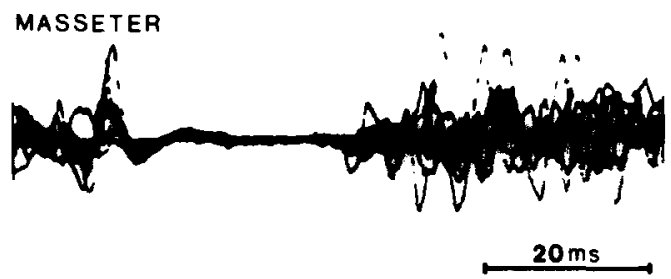

DIGASTRICUS

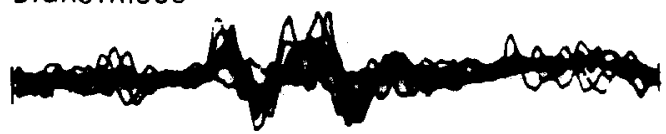

Fig. 3. Reflex activities after tap stimuli (10) on the chin during maximum clenching in edge-to-edge contact position. 
Tap on incisors during clenching in intercuspal edgeto-edge configuration

A clear jaw-opening reflex response was obtained when the tap was delivered to all four central incisors using a rubber stamp (Fig. 1). Using weak stimulation only, the short-latency response was elicited (Figs 4 and 5A). Its mean latency was $9.5+0.5 \mathrm{~ms}$, close to that of the jaw-jerk reflex in the masseter muscle $(8.5 \pm 0.5 \mathrm{~ms})$. Moderate stimuli elicited a second reflex component within the same record with a latency of about $20 \mathrm{~ms}$ (Fig. 5B). This was, however, not the case for all the subjects. Four subjects only demonstrated the long-latency response with no indication for the existence of an earlier reflex component. When strong, but not painful, stimuli were applied, the short-latency response disappeared in the digastric electromyogram in all subjects (Fig. 5C). The long-latency reflex activity occurred now $30 \mathrm{~ms}$ or more after stimulation of the incisors. This reflex activity showed less synchronization than the previously reported earlier reflex responses.

\section{Anaesthesia}

After anaesthesia, the jaw-opening response was depressed or abolished with all stimulus intensities (Fig. 6). The jaw-jerk reflex, however, remained intact.

\section{DISCUSSION}

Previously we showed a digastric muscle response in man elicited by a mechanical stimulation (Yamada and Ash, 1982) suggesting that the digastric muscle

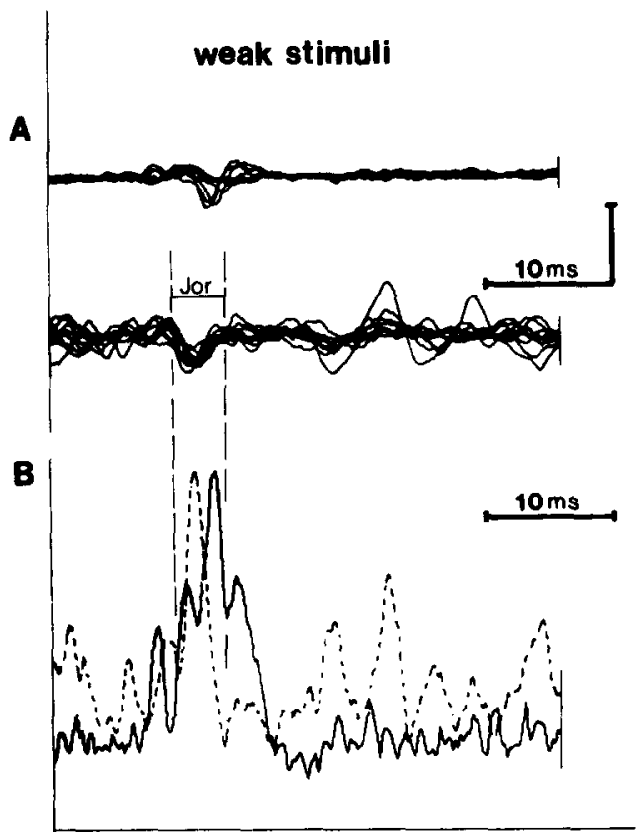

Fig. 4. Reflex activities after weak tap stimuli to all incisors during clenching in intercuspal edge-to-edge contact position. (A) Superimposition of 10 raw electromyograms of the masseter (upper) and digastric (lower) muscles. (B) Averaged and normalized response of 10 stimuli. The masster activity is indicated by smooth, the digastric by broken line. Calibrations: vertical bar equals 0.1 and $0.05 \mathrm{mV}$ for the masseter and digastric muscle, respectively.

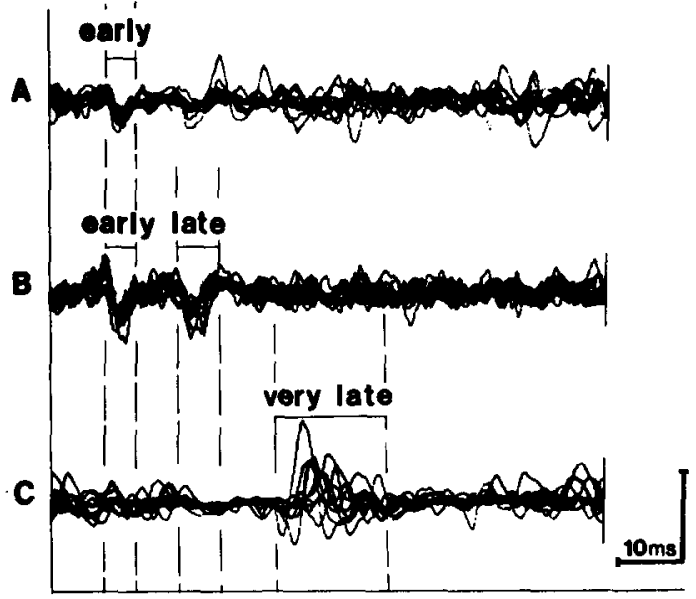

Fig. 5. Reflex activites of the digastric muscle obtained by different stimulus intensities. (A) Weak, (B) moderate and (C) strong (non painful) stimulation. Calibration: vertical bar equals $0.05 \mathrm{mV}$.

reflex tends to respond to low-threshold afferents and noxious stimuli may mask or inhibit the reflex. We suggested that moderate background activity in the depressor muscles is essential for this reflex. Clenching in incisal edge-to-edge contact position is associated with moderate background activity in the digastric muscle (Fig. 3); a clear digastric response was observed. Summation of the jaw-opening reflex response has been suggested in the case of animals (Anderson and Mahan, 1971; Schwaluk, 1971; Greenwood and Sessle, 1976). The incisal edge-to-

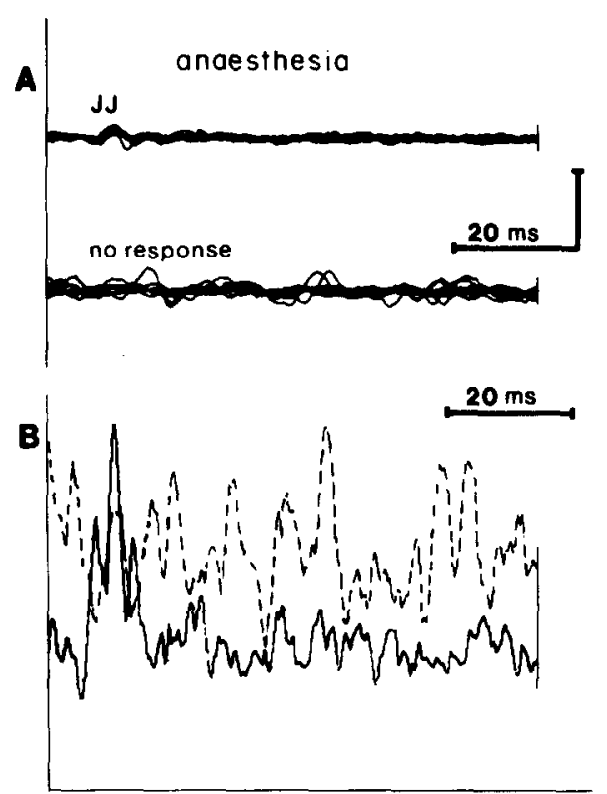

Fig. 6. Effect of local anaesthesia of upper and lower incisors. (A) Superimposition of 10 raw electromyograms of the masseter (upper) and digastric (lower) muscles. (B) Averaged and normalized response of 10 stimuli. The masseter activity is indicated by smooth, the digastric activity by broken line. Calibration: vertical bar equals $0.1 \mathrm{mV}$ for the masseter and $0.05 \mathrm{mV}$ for the digastric muscle. 
edge configuration has the advantage of allowing the simultaneous stimulation of all four central incisors. The short-latency reflex component was more often observed when the stimulus was applied to the incisors rather than the chin, suggesting that temporal summation may facilitate the early reflex component.

As studies in animals suggest that the jaw-opening reflex has at least a disynaptic pathway (Kikodoro et al., 1968; Sumino, 1971), the latency for the early reflex component was compared to the latency of the jaw-jerk response. For the jaw-jerk response, generally accepted as a monosynaptic pathway, a mean latency of $8.5 \mathrm{~ms}$ was found. One millisecond, however, is not enough to account for another synaptic transmission. Fujii (1977) has found that there is about $1 \mathrm{~ms}$ difference in latency between a $\mathrm{T}$-wave elicited by a jaw-jerk reflex and a $\mathrm{H}$-wave elicited by electrical stimulation. He then suggested that there must be at least $1 \mathrm{~ms}$ delay until the firing of the muscle spindles due to the time needed for the propagation of the vibration stimulus from the chin to the muscle spindles of the masseter. Direct stimulation of the incisors is undoubtedly faster. This would leave about $2 \mathrm{~ms}$ for the assumed second synaptic delay.

The relationship we found between stimulus intensity and digastric response is in conflict with that reported by Keller et al. (1972) and Munakata (1981) from experiments in cats in which high-threshold afferents were associated with the digastric response. Munakata (1981) claimed that the short-latency response (termed early response) was due to excitation of high-threshold and the long-latency response (late response) was related to lower-threshold afferents. Our study in man, however, shows that the shortlatency response may be elicited by a weak stimulus. Strong stimuli elicited a long-latency response with less synchonization than the earlier responses. Dickenson, Le Bars and Besson (1980) showed that noxious stimuli may depress the activity of neurones within the trigeminal sensory nucleus. Other findings support this; electrical or noxious stimulation in man do not elicit any opening response in the digastric muscle (Yemm, 1972; Matthews, 1975). These different findings may be due to differences in experimental conditions. Sessle (1977b) suggested that the corticofugal inhibitory influence on the digastric reflex is somatotopically organized. It is possible that the human cortex, in some circumstances, inhibits the reflex. Under certain circumstances such as the protrusive position employed here, the inhibitory effect may be less.

Because in our experiments the response was greater when the incisors were mechanically stimulated and local anaesthesia of the incisors diminished or abolished the reflex response, we conclude that mechanoreceptors of the periodontal apparatus are involved in this jaw-opening reflex pathway.

\section{REFERENCES}

Anderson K. V. and Mahan P. E. (1971) Interaction of tooth pulp and periodontal ligament receptors in a jawdepression reflex. Expl Neurol. 32, 295-302.

Beaudreau D. E., Daugherty W. R. and Masland W. S.
(1969) Two types of motor pause in masticatory muscles. Am. J. Physiol. 216, 16-21.

Dickenson A. H., Le Bars D. and Besson J. M. (1980) Diffuse noxious inhibitory controls (DNIC). Effects on trigeminal nucleus caudalis neurones in the rat. Brain Res. 200, 293-305.

Fujii H. (1977) Evoked EMG of masseter and temporal muscles in man. $J$, oral Rehab. 4, 291-303.

Gillings B. R. D. and Klineberg I. J. (1975) Latency and inhibition of human masticatory muscles following stimuli. J. dent. Res. 54, 260-279.

Greenwood L. F. and Sessle B. J. (1976) Pain, brain stem mechanisms, and motorfunction. In: Mastication and Swallowing: Biological and Clinical Correlates (Edited by Sessle B. J. and Hannam A. G.) pp. 108 119. University of Toronto Press, Toronto, Canada.

Hannam A. G., Matthews B. and Yemm R. (1968) The unloading reflex in masticatory muscles of man. Archs oral Biol. 13, 361-364.

Hannam A. G. and Matthews B. (1969) Reflex jaw opening in response to stimulation of periodontal mechanoreceptors in the cat. Archs oral Biol. 14, 415-419.

Hannam A. G. and Lund J. P. (1981) The effect of intra-oral stimulation on the human masticatory cycle. Archs oral Biol. 26, 865-870.

Junge D., Faermark W. and Friedman D. H. (1979) Bilaterality of the jaw-opening reflex in the cat. Archs oral Biol. 24, 21-25

Keller O., Vyklicky L. and Sykova E. (1972) Reflexes from A-delta and A-alpha trigeminal afferent. Brain Res. 37, 330-332.

Kidokoro Y., Kubota K., Shuto S. and Sumino R. (1968) Reflex organization of cat masticatory muscles. J. Neurophysiol. 31, 695-708.

Matthews B. (1975) Mastication. In: Applied Physiology of the Mouth (Edited by Lavelle C. L. B.) pp. 199-242. Wright, Bristol.

Munakata Y. (1981) Neurophysiological studies on the mechanisms of two different responses in the jaw opening reflex of cats. Jap. J. oral Biol. 23, 534-547.

Nakamura Y., Katoh M., Enomoto S. and Hiraba K. (1982) Neural pathway involved in the excitation of motoneurones to jaw-opening muscles by stimulation of the orbital cerebral cortex in the cat. Archs oral Biol. 27, 283-287.

Schwaluk S. (1971) Initiation of reflex activity from the temporomandibular joint of the cat. $J$. dent. Res. 50 , $1642-1646$

Sessie B. J. and Greenwood L. F. (1976) Role of trigeminal nucleus caudalis in the modulation of trigeminal sensory and motor neuronal activities. In: Advances in Pain Research and Therapy (Edited by Bonica J. J. and AlbeFessard D.) pp. 185-189. Raven, New York.

Sessle B. J. (1977a) Modulation of alpha and gamma trigeminal motoneurons by various peripheral stimuli. Expl Neurol. 54, 323-339.

Sessle B. J. (1977b) Identification of alpha and gamma trigcminal motoncurones and effects of stimulation of amygdala, cerebellum, and cerebral cortex. Expl Neurol. 54, 303-322.

Sumino R. (1971) Central neural pathways involved in the jaw opening reflex in the cat. In: Oral-Facial Sensory and Motor Mechanisms (Edited by Dubner R. and Kawamura Y.) pp. 315-322. Appleton-Century-Crofts, New York.

Thexton A. J. (1974) Jaw opening and closing reflexes in the cat. Brain Res. 66, 425-433.

Yamada Y. and Ash M. M. Jr (1982) An electromyographic study of jaw opening and closing reflexes in man. Archs oral Biol. 27, 13-19.

Yemm R. (1972) Reflex jaw opening following electrical stimulation of oral mucous membrane in man. Archs nral Biol. 17. 513-523. 